\title{
Understanding the birefringence effects in an all-fiber device based on photonic crystal fibers with integrated electrodes
}

\author{
Erick Reyes-Vera, Esteban González-Valencia, and Pedro Torres ${ }^{*}$ \\ School of Physics, National University of Colombia, Medellín campus, A.A. 3840, Medellín, Colombia
}

Received November 15, 2010; accepted November 27, 2010; published December 31, 2010

\begin{abstract}
In this work, we study a special kind of photonic crystal fibers with two integrated electrodes. The device operation is based on induced birefringence caused by mechanical stress when electrical current heats the internal electrodes. Due to the complex structure of the fiber, a finite element model was carried out to determine the strain distributions generated at the center region of the fiber core, and hence taking the induced change in the refractive index as a change in the effective refractive index due to the transferred heat. Here, we explain the interesting result that fiber birefringence axes rotated asymptotically with temperature. The analysis shows that this rotation of the birefringence axes is the cause of a nonlinear variation of the effective refractive index of the fundamental mode.
\end{abstract}

Photonic crystal fibers (PCFs), also called microstructured optical fibers (MOFs) or holey fibers (HFs), are waveguides that contain an array of air holes running along the length of the fiber. The air-hole lattice structure provides an additional design freedom offering different light propagation properties, including endlessly single-mode operation, very large or small effective core area, and scalable dispersion [1]. In general, PCFs guide light either by a total internal reflection (TIR) or photonic band gap (PBG) effect, depending on the fiber structure.

The potential applications of PCFs can be further broadened using post-processing. One possibility is the insertion, within the microstructure, of a wide variety of materials. The typical materials include temperaturedependent polymers [2] and liquid crystals [3-4], in which the optical properties are tuned by heating or by the application of an external field (electric or magnetic), respectively, to the PCF system. The development of these special optical devices opens new possibilities because they allow broad control of fiber optical parameters. Nonetheless, this approach does not explore the possibility of creating all-fiber devices.

A special kind of silica PCF containing two integrated electrodes to actively control the spatial distribution of a refractive index was demonstrated by passing a current pulse through one electrode [5]. Fig. 1 shows the cross section of this PCF with both external holes filled with 43Bi-57Sn alloy that occupied the entire cross-section of the holes. The microstructure consists of five rings of air

\footnotetext{
"E-mail: pitorres@unal.edu.co
}

holes with a diameter of $1.5 \mu \mathrm{m}$ and a $2.5-\mu \mathrm{m}$ diameter solid core; the average pitch is $2.2 \mu \mathrm{m}$. When applying the current pulse to the electrode, this is heated by Joule effect causing it to expand rapidly and inducing thermomechanical stress on the fiber structure.

Dynamic measurements were carried out by electrically connecting one of the electrodes and the electro-optical characterization was performed for an input beam linearly polarized and a fixed analyzer was used to transform polarization rotation into amplitude modulation [2]. The variation of the signal amplitude with the applied current indicates that the induced birefringence in the fiber is proportional to the heat deposited in the metal electrode. Subsequent measurements by applying a steady (dc) current for several seconds show that when the electric current is switched on the, birefringence of the fiber is modified and so is the state of polarization of the exiting beam.

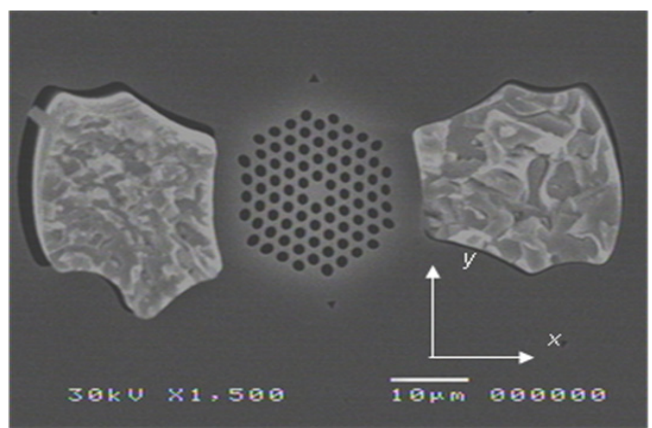

Fig. 1. SEM image of the silica PCF with both external holes filled with 43Bi-57Sn alloy.

We have recently accommodated the PCF with integrated electrodes on a Peltier element as an alternative to make a device with two active electrodes [6], so when heat is transferred to the PCF, the internal electrodes of the fiber will expand and modify the strain distribution of the squeezed region. The experimental characterization was realized by coupling the linearly polarized beam of a diode laser at $1550 \mathrm{~nm}$ to the PCF, and the light exiting the fiber passes through a rotating analyzer before 
reaching the detector, so that the output power measured was a function of the orientation of the birefringent axes $\phi$ and the total retardation $\Psi$. In the case of uniform strain over a fiber length $L, \Psi=B_{\mathrm{T}} k_{0} L$ where $B_{\mathrm{T}}$ is the magnitude of the resultant birefringence and $k_{0}$ the wave number in the vacuum. The fiber was around $10.7 \mathrm{~cm}$ in length, including $4 \mathrm{~cm}$ that were located on the Peltier element so that the two electrodes are at the same height to ensure uniform thermal conditions. The resolution in the rotating analyzer was $5^{\circ}$. Here, unlike the previous analysis, the variation of the signal amplitude is measured at constant temperature (static measurements) without any electrical load on the internal electrodes of the fiber. We found an interesting result that the birefringence axes rotated asymptotically with temperature. The analysis shows that this rotation of the birefringence axes is the cause of the nonlinear variation of an effective refractive index of the fundamental mode.

To understand the mechanism by which the device operates, we use a finite element (FE) approach to analyze the birefringence induced by the expansion of the electrodes within the fiber at a given temperature.

Due to the insertion of the electrodes, the PCF presents a residual strain distribution responsible for the intrinsic birefringence. When heat is transferred to the PCF, the internal electrodes of the fiber will expand and modify the strain distribution. FE simulations of the PCF were carried out to determine the strain distributions generated in the fiber. This method allows determining the strain components $\varepsilon_{\mathrm{x}}$, and $\varepsilon_{\mathrm{y}}$ about the optical axes $x$ and $y$ (see Fig. 1) and the shear-strain component $\gamma_{\mathrm{xy}}$ by meshing the fiber cross section and solving the equations that represent the relationships among the stress, strain and other thermo-elastic variables. The principal strain components in the squeezed fiber section are determined using Mohr's circle theory [7]:

$$
\varepsilon_{1,2}=\frac{\varepsilon_{x}+\varepsilon_{y}}{2} \pm \sqrt{\left(\frac{\varepsilon_{x}-\varepsilon_{y}}{2}\right)^{2}+\left(\frac{\gamma_{x y}}{2}\right)^{2}} .
$$

In addition, the angle of orientation of the optical propagation axes $\phi$ is given by

$$
\tan 2 \phi=\frac{\gamma_{x y}}{\varepsilon_{x}-\varepsilon_{y}} .
$$

Then, the rotation of the birefringent axes is a function of temperature.

Figure 2(a) shows the variation of average principal strains $\varepsilon_{1}$ and $\varepsilon_{2}$ over a given core area with temperature.
It gives a good understanding of how the all-fiber device operates. We can see that the thermo-elastic response of the fiber core to temperature is nonlinear. We found that the nonlinearity is due to the rotation, described by Eq. (2), of the principal axes with respect to the initial polarization axes. The values of $\phi$ depending on temperature, taking as reference the result for $\mathrm{T}=26^{\circ} \mathrm{C}$, are shown in Fig. 2(b). Clearly, we can see that the experimental results reported in [6] confirm, within the uncertainty in the analyzer angle, the simulation results. Here, the negative sign of rotation indicates that the effect is clockwise.
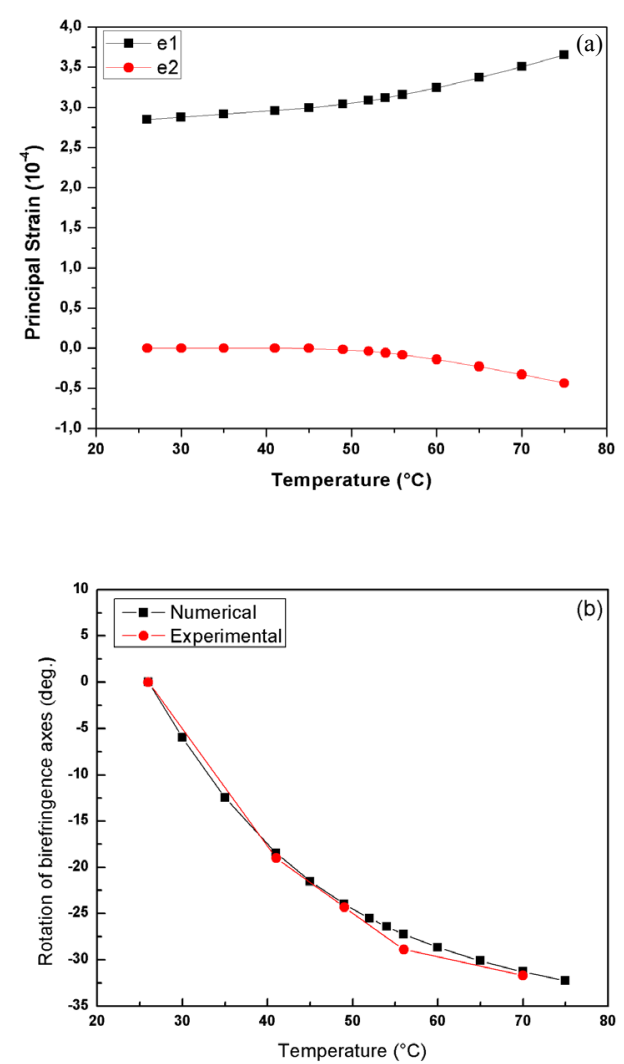

Fig. 2. (a) Average principal strains over a given PCF core area as a function of the temperature in the PCF with integrated electrodes. (b) Comparison between numerical and experimental [6] results of the rotation of birefringence axes.

On the other hand, changes in the effective refractive index associated with the optical propagation axes can be evaluated without the modal calculation even for photonic crystal fiber [8], by using Eq. (3), where $n_{\mathrm{eff}, i}$ is the effective refractive index along the optical propagation axis $i ; p_{11}$ and $p_{12}$ are the strain-optic coefficients. 


$$
\begin{aligned}
& \Delta n_{1}=-\frac{n_{\mathrm{eff}, 1}^{3}}{2}\left(p_{11} \varepsilon_{1}+p_{12} \varepsilon_{2}\right) \\
& \Delta n_{2}=-\frac{n_{\mathrm{eff}, 2}^{3}}{2}\left(p_{11} \varepsilon_{2}+p_{12} \varepsilon_{1}\right) .
\end{aligned}
$$

Figure 3 shows simulation results for the refractive index change and the magnitude of the induced birefringence. One notes the nonlinear variation of the refractive index change, thus the resulting induced birefringence, with temperature. This nonlinearity is consistent with previously published experimental results in conventional fibers with internal electrodes [9, 10].
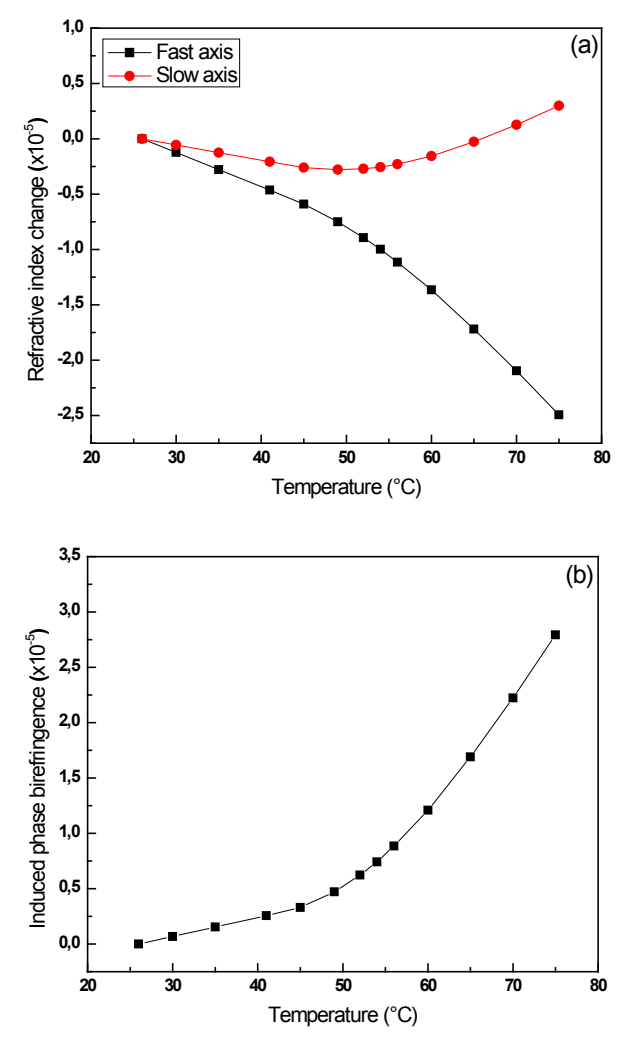

Fig. 3. (a) Effective refractive index change and (b) induced phase birefringence at $1556 \mathrm{~nm}$ as a function of temperature in the allfiber device.
In summary, understanding the effect of stress inducing metals on the optical characteristics of birefringent sidehole fiber is a very important step to create all-fiber electro-optical devices. In this work bismuth-tin alloy has been studied as s stress inducing material. Other metal alloys will be studied, especially indium, due its large thermal expansion coefficient [10].

This work was supported by the National University of Colombia through the Bicentenario program under Grant 90201022. E. Reyes-Vera thanks Colciencias and the National University of Colombia for supporting his scholarship.

\section{References}

[1] P.St.J. Russell, J. Lightwave Technol. 24, 4729 (2006).

[2] B.J. Eggleton, C. Kerbage, P.S. Westbrook, R.S. Windeler, A. Hale, Opt. Exp. 9, 698 (2001), http://www.opticsinfobase.org/oe/ abstract.cfm?URI=oe-9-13-698.

[3] T.T. Larsen, A. Bjarklev, D.S. Hermann, J. Broeng, Opt. Exp. 11, 2589 (2003), http://www.opticsinfobase.org/oe/abstract.cfm? URI=oe-11-20-2589.

[4] F. Du, Y.-Q. Lu, and S.-T. Wu, Appl. Phys. Lett. 85, 2181 (2004).

[5] G. Chesini et al., Opt. Exp. 17, 1660 (2009), http:// www.opticsinfobase.org/oe/abstract.cfm?URI=0e-17-3-1660.

[6] E. Reyes-Vera et al. in Latin America Optics and Photonics Conference (LAOP) (Washington, Optical Society of America 2010), ThF2.

[7] S.P. Timoshenko, J.N. Goodier, Theory of Elasticity, 3rd ed. (New York, McGraw-Hill 1970).

[8] J.F. Botero-Cadavid, J.D. Causado-Buelvas, P. Torres, J. Lightwave Technol. 28, 1291 (2010).

[9] Z. Yu, O. Tarasenko, W. Margulis, and P. -Y. Fonjallaz, Opt. Exp. 16, 8229 (2008), http://www.opticsinfobase.org/oe/abstract.cfm? URI=oe-16-11-8229.

[10] S.H. Lee, B.H. Kim, W.-T. Han, Opt. Exp. 17, 9712-9717 (2009), http://www.opticsinfobase.org/oe/abstract.cfm?URI=oe-17-12-9712. 